\title{
Aspects Tomodensitometriques des Infections Rachidiennes dans le Service d'Imagerie Medicale du CNHU-HKM a Cotonou (Benin)
}

\author{
Adjadohoun Sonia, Bignon Mahussi Gwladys (MA Radiologie) \\ Yekpe Ahouansou Patricia (MCA Radiologie) \\ Ngamo Gabriel (DES de Radiolgie) \\ Kuye-Ndongo Brigitte (DES de Radiologie) \\ Baglo Anita (DES de Radiologie) \\ Université d'Abomey-Calavi, \\ Faculté des Sciences de la Santé de Cotonou, Bénin \\ Savi De Tove Kofi-Mensa (MCA Radiologie)
}

Université de Parakou, Faculté des Sciences de la santé de Parakou, Bénin

Zomalheto Zavier (PT Rhumatologie)

Biaou Olivier (PT Radiologie)

Université d'Abomey-Calavi,

Faculté des Sciences de la Santé de Cotonou, Bénin

Doi:10.19044/esj.2021.v17n17p350

Submitted: 14 October 2020

Accepted: 13 April 2021

Published: 31 May 2021
Copyright 2021 Author(s)

Under Creative Commons BY-NC-ND

4.0 OPEN ACCESS

Cite As:

Adjadohoun SBGM, Yekpe Ahouansou P., Ngamo G., Kuye-Ndongo B., Baglo A., Savi De Tove K-M., Zomalheto Z. \& Biaou O. (2021). Aspects Tomodensitometriques des Infections Rachidiennes dans le Service d'Imagerie Medicale du CNHU-HKM a Cotonou (Benin). European Scientific Journal, ESJ, 17(17), 350. https://doi.org/10.19044/esj.2021.v17n17p350

\section{Résumé}

Introduction : Les atteintes infectieuses du rachis (AIR) sont rares, mais en recrudescence en raison des affections immunodépressives et immunosuppressives. Elles représentent 4 à $7 \%$ de toutes les ostéomyélites. $\mathrm{Au}$ Centre National Hospitalier Universitaire Hubert Koutoukou Maga (CNHU HKM) de Cotonou (Bénin), elles constituent 2,31\% des hospitalisations. L'objectif de ce travail était de décrire leurs caractéristiques tomodensitométriques. Matériels et méthode : Étude transversale descriptive à collecte rétrospective menée dans le service d'imagerie médicale du CNHUHKM sur une période de six (06) ans allant du 1er Janvier 2012 au 31 
Décembre 2017. Portant sur les comptes rendus de scanner où le diagnostic d'AIR avait été retenu. Les examens tomodensitométriques avaient été réalisés à l'aide d'un Scanner Siemens Somatom Emotion ${ }^{\circledR}$ (16 barrettes modèle 2011). Résultats : Sur 2797 scanners du rachis, 140 AIR (3,5\%) avaient été suspectées et $72(2.6 \%)$ avaient été confirmées sur les images scanographiques. L'âge moyen était de 49,98 ans $\pm 15,5$ ans avec une prédominance masculine (sex ratio $=1,2)$. Le segment de prédilection était lombaire $(77,8 \%)$ avec des érosions en miroir $(11,12 \%)$ principalement au niveau de l'étage L3-L4 (12.5\%). Une hypodensité discale était retrouvée dans $16.7 \%$ des cas. L'atteinte somatique la plus fréquente était l'ostéocondensation (20,8\%). Les tassements vertébraux étaient observés dans $9.72 \%$ et les listhésis dans $11.11 \%$ des cas. Les diagnostics de discite, de spondylite, et de spondylodiscite avaient été retenus respectivement dans $6,94 \%, 8,33 \%$ et $84,72 \%$ des cas. Une extension aux parties molles était retrouvée dans 27,8 \% des cas dont un abcès des parties molles dans 22,22\% des cas. Conclusion : Au décours de cette étude, le diagnostic des AIR bien que tardif, a majoritairement été posé au scanner avant la survenue de complications. Les érosions en miroir des spondyles étaient fortement évocatrices en dehors de toute hypodensité discale.

Mots clés : Rachis, infections, scanner Cotonou 


\title{
Tomodensitometric Aspects of Spinal Infections in the Medical Imaging Department of the Cnhu-Hkm of Cotonou (Benin)
}

\author{
Adjadohoun Sonia, Bignon Mahussi Gwladys (MA Radiologie) \\ Yekpe Ahouansou Patricia (MCA Radiologie) \\ Ngamo Gabriel (DES de Radiolgie) \\ Kuye-Ndongo Brigitte (DES de Radiologie) \\ Baglo Anita (DES de Radiologie) \\ Université d'Abomey-Calavi, \\ Faculté des Sciences de la Santé de Cotonou, Bénin \\ Savi De Tove Kofi-Mensa (MCA Radiologie)
}

Université de Parakou, Faculté des Sciences de la santé de Parakou, Bénin

\section{Zomalheto Zavier (PT Rhumatologie) \\ Biaou Olivier (PT Radiologie) \\ Université d'Abomey-Calavi,}

Faculté des Sciences de la Santé de Cotonou, Bénin

\begin{abstract}
Introduction: Infections of the spine (AIR) are rare but increase because of immunosuppressive and immunosuppressive disorders. They represent 4 to $7 \%$ of all osteomyelitis. At the Hubert Koutoukou Maga National University Hospital Center (CNHU HKM), they constitute $2.31 \%$ of hospitalizations. The aim of this work is to describe their CT characteristics at the CNHU-HKM in Cotonou. Material and method: Retrospective descriptive cross-sectional study conducted of the CNHU-HKM medical imaging department over a period of 06 years from January 1, 2012 to December 31, 2017. Included were all CT scan reports where the diagnosis was made AIR had been retained. Computed tomographic examinations were performed using a Siemens Somatom Emotion ${ }^{\circledR}$ Scanner (16 slices model 2011). Results: Of 2,797 spine scans, 140 IRAs were suspected and 72 (2.6\%) were diagnosed on the scanographic images. The average age was $49.98 \pm 15.5$ years with a male predominance $($ sex ratio $=1.2)$. The preferred segment was lumbar (77.8\%) with mirror erosions (11.12\%) mainly at the L3-L4 stage (12.5\%). A disc hypodensity was found in $16.7 \%$ of cases. The most common somatic involvement was osteocondensation (20.8\%). Vertebral compression was observed in $9.72 \%$ and listhesis in $11.11 \%$ of cases. Diagnoses of discitis, spondylitis, and spondylodiscitis were retained in $6.94 \%, 8.33 \%$ and $84.72 \%$ of cases, respectively. Soft tissue extension was found in $27.8 \%$ of cases including soft tissue abscess in $22.22 \%$ of cases. Conclusion: During the
\end{abstract}


course of this study, the diagnosis of RIA, although late, was mainly performed on CT before the occurrence of complications. The mirrored erosions of the spondyls were strongly evocative outside of any disc hypodensity.

Keywords: Spine, Infection, CT, Cotonou

\section{Introduction}

Les atteintes infectieuses du rachis (AIR) sont rares, mais en recrudescence en raison des affections immunodépressives et immunosuppressives. Elles représentent de 4 à $7 \%$ de toutes les ostéomyélites (L.Badaoui, G.Dabo, M.Sodqi, L.Marih, A.Oulad lahsen, A.Chakib, 2014). Les AIR peuvent concerner n'importe quelle composante de la colonne vertébrale : du corps vertébral, disque intervertébral à l'arc postérieur et peuvent se compliquer par une extension aux tissus mous para-spinaux, à l'espace épidural et au canal rachidien(Tyrrell P \& Mccall, 1999). En Afrique, le bacille de $\mathrm{KOCH}$ et le staphylocoque doré sont les germes principalement incriminés (L.Badaoui, G.Dabo, M.Sodqi, L.Marih, A.Oulad lahsen, A.Chakib, 2014). Au CNHU HKM, les AIR représentent 2,31\% des hospitalisations et le diagnostic repose souvent sur un triple argument clinique, radiologique et bactériologique (Zomalheto et al., 2015). La radiologie en particulier l'IRM reste l'examen de choix dans le diagnostic précoce de l'atteinte rachidienne. Cependant son coût et son accessibilité limitent sa réalisation dans la plupart des pays africains en particulier le Bénin où la tomodensitométrie rachidienne reste l'examen le plus pratiqué. L'objectif de ce travail a été donc de décrire les caractéristiques tomodensitométriques des AIR au CNHU HKM de Cotonou.

\section{Matériels et méthode}

Cette étude a été réalisée dans le service d'Imagerie Médicale du CNHU HKM de Cotonou au Sud Benin. Il s'est agi d'une étude transversale, descriptive à collecte rétrospective des données sur une période de 6 ans de Janvier 2012 à Décembre 2017. Nous avons colligé tous les comptes rendus de scanner du rachis, délivrés sur la période d'étude où le diagnostic d'AIR avait été retenu. Les examens TDM avaient été réalisés à l'aide d'un Scanner Siemens Somatom Emotion ${ }^{\circledR}$ (16 barettes modèle 2011). Tous les patients avaient été explorés par une acquisition hélicoïdale rachidienne en coupes fines de $1 \mathrm{~mm}$ d'épaisseur, sans et avec injection de produit de contraste iodé. Des reconstructions multi planaires en fenêtre osseuse et parties molles et tridimensionnelles ont été réalisées à l'aide des logiciels: efilms WorkSation ${ }^{\circledR}$, Radiant Dicoms viewer® 4.6.2 et Horos Dicoms viewer ${ }^{\circledR}$. Les 
interprétations ont été faites par un médecin radiologue. Les critères diagnostic étaient les suivants:

- Spondylite: Ostéolyse (lésions érosives des plateaux), ostéocondensation, mixte ;

- Discite : Hypodensité, pincement, collection ;

- Parties molles : Epaississement, collections, calcifications ;

Les données radiologiques étaient exploitées dans un contexte clinique (altération de l'état général et le syndrome rachidien) et biologiques (hémocultures, prélèvement aux portes d'entrées, PCR, IDR quantiferon) pour le diagnostic d'AIR. Aucune biopsie n'a été faite.

Les variables utilisées étaient les variables sociodémographiques (le sexe, l'âge) ; les motifs d'examen (arguments cliniques), les caractéristiques sémiologiques: localisation (cervicale, thoracique, lombaire, sacrée), nombres de vertèbres atteintes, composants atteints (corps vertébral, disque, parties molles), lésions élémentaires (lyse, condensation ou mixte, érosion, tassements et listhésis), collection pré et para vertébrale ; les extensions (para vertébrale, pré vertébrale, épidurale, arc postérieur) ; les infiltrations (abcès, calcifications); les complications à type de troubles de la statique et de compression médullaire.

Le traitement et l'analyse des données ont été informatiques. Les données des comptes rendus ont été encodées dans un le logiciel Microsoft Office Excel $2016{ }^{\circledR}$ et traitées à l'aide du logiciel Epi info 7.1.

\section{Résultats}

\section{Caractéristiques épidémiologiques}

Sur 2797 TDM du rachis, 140 AIR (3,5\%) avaient été suspectées cliniquement, $72(2,6 \%)$ atteintes infectieuses ont été diagnostiquées. La figure 1 présente la fréquence des cas en fonction des années.

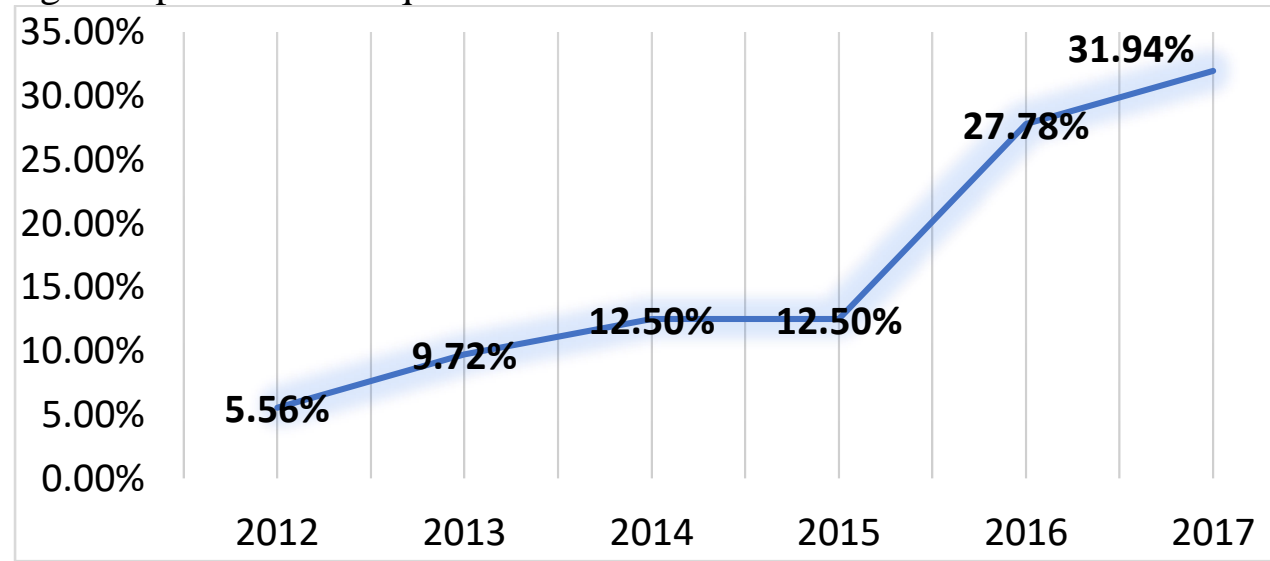

Figure : 1: Fréquence des cas en fonction des années 
L'âge moyen était de 49,98 ans $\pm 15,5$ ans. Le sexe masculin était le plus atteint avec une sex ratio de 1,2 .

\section{Caractéristiques tomodensitométriques \\ Siège}

Le segment de prédilection était lombaire $(77,8 \%)$ suivi du segment thoracique $(6,67 \%)$, cervical $(2,78 \%)$ et sacré (1.39\%) (Figure 2).

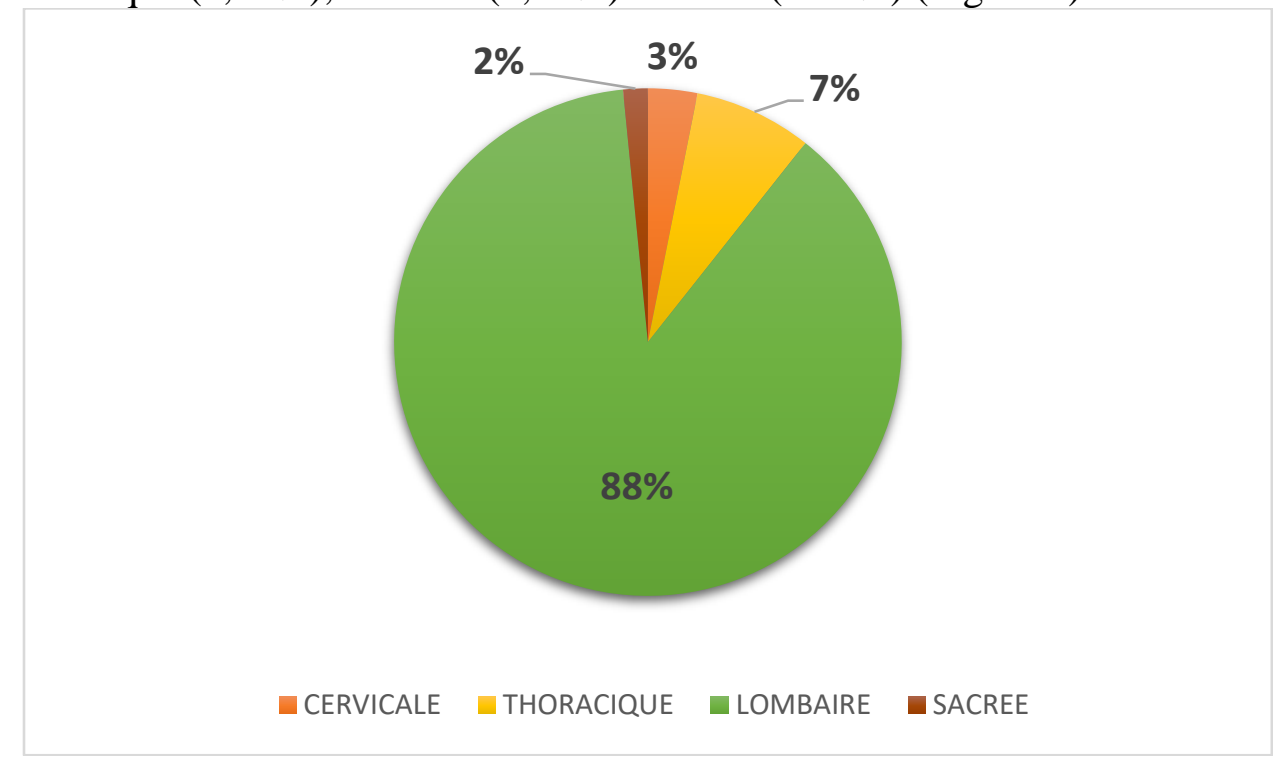

Figure 2 : Répartition des AIR en fonction du segment atteint

Nous avons retrouvé une atteinte contiguë des vertèbres chez 93,04\% des patients (bi-vertébrale chez 10,38\%, trois vertèbres chez 5,55\% et plus de trois vertèbres chez 73,61\%). L'atteinte était uni vertébrale dans 6,94\%. (Tableau I).

Tableau I : Répartition des AIR par étages vertébraux

\begin{tabular}{lc}
\hline Atteintes & Pourcentage (\%) \\
\hline Contiguë & 93,04 \\
Bi-vertébrale & 10,38 \\
Trois vertèbres & 05,55 \\
Plus de trois vertèbres & 73,61 \\
Uni vertébrale & $6,94$. \\
\hline
\end{tabular}

\section{Nature des lésions}

Une discite était retrouvée dans $8 \%$ des cas, une spondylite dans $7 \%$ et une spondylodiscite dans $85 \%$. La figure 3 représente la répartition des AIR selon l'âge des patients. 


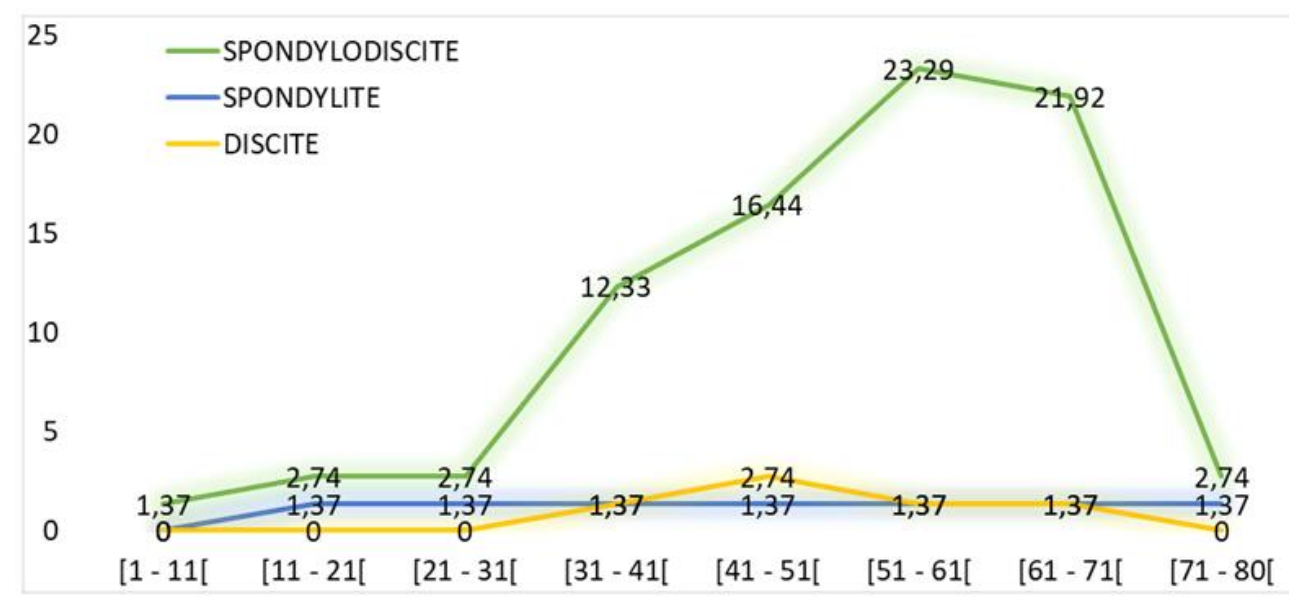

Figure 3 : Répartition des AIR selon l'âge des patients

\section{Lésions osseuses}

Sur le plan sémiologique, les érosions des plateaux vertébraux étaient retrouvées dans la quasi-totalité des cas dans notre série $(94,44 \%)$. Des érosions en miroir étaient notées dans $38,88 \%$ des cas principalement au niveau de l'étage L3-L4 (12.5\%). Les tassements vertébraux étaient retrouvés dans $9,72 \%$ des cas. La figure 4 représente une reconstruction sagittale en fenêtre osseuse d'un rachis lombaire présentant les atteintes somatiques fréquemment retrouvés dans notre série.

Les lésions de type infectieuse étaient associées à des remaniements osseux dégénératifs dans $44,44 \%$ avec une prédominance de l'ostéocondensation (20,83\%) suivie de la lyse osseuse dans $16,67 \%$. Les lésions étaient mixtes dans 6,94\% des cas. La figure 4 illustre une association des deux types de lésions.

\section{Lésions discales}

L'atteinte discale était dominée par les pincements retrouvée dans $66,67 \%$ suivit des hypodensités discales dans $16,7 \%$ des cas. Les collections des disques étaient rares $1,39 \%$. La figure 5 présente des reconstructions sagittale et axiale en fenêtre discale des principales lésions des disques retrouvées dans notre série.

\section{Extensions}

Les extensions des lésions en para vertébrale étaient retrouvées dans $18,06 \%$, en pré vertébrale dans $11,11 \%$ en épidurale dans $4,17 \%$, à l'arc postérieur dans $2,8 \%$ et aux parties molles dans $27,8 \%$. 


\section{Complications}

Les complications avaient été retrouvées dans $37,5 \%$ des dossiers. Le tableau II illustre ces complications. Elles étaient dominées par les abcès des parties molles para vertébrales $(22,22 \%)$ suivis des abcès épiduraux $(4,17 \%)$, les calcifications des parties molles dans $8,3 \%$, les troubles de la statique à type de cyphose $(6,94 \%)$, de bloc osseux $(6,94 \%)$ et de scoliose $(1,39 \%)$. Les compressions médullaires représentaient $9,72 \%$ des complications.

Tableau II : Répartition des complications des AIR

\begin{tabular}{lc}
\hline COMPLICATIONS & Pourcentage $\mathbf{( \% )}$ \\
\hline Abcès des parties molles para vertébrales & 22,22 \\
\hline Abcès épiduraux & 04,17 \\
\hline Calcifications des parties molles & 08,3 \\
\hline Troubles de la statique & \\
\hline \multicolumn{1}{c}{ Cyphose } & 06,94 \\
\hline \multicolumn{1}{c}{ Scoliose } & 01,39 \\
\hline$\quad$ Bloc osseux & 06,94 \\
\hline Compressions médullaires & 09,72 \\
\hline
\end{tabular}

\section{Discussion}

La confirmation d'une atteinte infectieuse du rachis est anatomopathologique avec la mise en évidence sur le prélèvement osseux par biopsie scanner guidée ce qui n'a pas été le cas sur la totalité des dossiers étudiés.

Nous avions retrouvé 2,6\% des AIR similaire aux résultats de Zomalheto et al en 2015 (Zomalheto et al., 2015) à Cotonou et ceux de Badoui et al en Tunisie en 2014 ( 2 à 4\%) (L.Badaoui, G.Dabo, M.Sodqi, L.Marih, A.Oulad lahsen, A.Chakib, 2014). Les sujets les plus atteints étaient les sujets proches de la cinquantaine comme l'avait retrouvé Kumar et al aux USA en 2017(Kumar et al., 2017).

La prédominance masculine observée est conforme aux données de la littérature en Afrique Sub-saharienne avec une sex ratio homme /femme de 1,2 comme retrouvé par Lamini et al au Congo $(1,3)$ (Lamini, 2019)et GbanéKoné $\mathrm{M}$ et al en Côte d'Ivoire $(1,16)$ (Gbané-Koné et al., 2015). Le contexte endémique, la recrudescence des pathologies immunodéficientes, les conditions socioéconomiques défavorables (précarité, promiscuité, mauvaise hygiène de vie) sont autant de facteurs favorisant l'infection dans nos pays(Annabi et al., 2008).

Il existait une augmentation progressive au cours des années des cas de AIR diagnostiqués à la TDM dans le service sur 6 ans. Gbané-Koné M en Côte d'Ivoire avait noté que le taux de réalisation(Gbané-Koné et al., 2015) de la TDM avait doublé en une décennie dans le service de Rhumatologie du CHU de Cocody(Gbané-Koné et al., 2015). Cette croissance de la prescription et de la réalisation des TDM pour AIR pourrait être dû à une 
meilleure accessibilité à la TDM, l'augmentation du nombre de spécialistes et des consultations spécialisées, également à l'augmentation de la fréquence hospitalière comme le rapporte Zomalheto et al dans leurs travaux à Cotonou en 2015(Zomalheto et al., 2015).

Sur le plan sémiologique tomodensitométrique, du point de vue topographique l'étage lombaire était le plus souvent atteint comme le corroborent Thouvenin et al en France en 2010 (Y. Thouvenin \& Cyteval, 2010) et Gbané-Koné M en Côte d'Ivoire(Gbané-Koné et al., 2015). L'atteinte localisée à un seul étage prédominait comme l'a retrouvé Gbané-Koné $\mathrm{M}$ en Côte d'Ivoire(Gbané-Koné et al., 2015).

Concernant l'atteinte des spondyles, Nous avions retrouvé un remodelage osseux dans 20,83\% contrairement aux études faites au Maroc où le diagnostic était posé à une phase précoce marquée par l'ostéolyse(Khalil et al., 2007). Ce qui amène à reposer l'épineux problème de la consultation tardive des patients dans notre contexte de pays en développement. Le retard diagnostique fait l'unanimité des auteurs(Toloba Y, Diallo S, Maiga Y, Sissoko BF, Ouattara K, Soumare D, 2011; Y. Thouvenin \& Cyteval, 2010). Dans notre série, les patients ont réalisé un scanner au stade de complications (37,5\% des dossiers) et $8,33 \%$ avec collections abcédées évoluées vers des calcifications. Elles ont été retrouvées au niveau des parties molles dans 26,39\% des cas ce qui corrobore les résultats de Alliez et al en France en 2015 (Bouillot et al., 2015).

Dans notre série aucun patient n'avait réalisé une imagerie par résonnance magnétique (IRM). L'IRM à une meilleure sensibilité pour le diagnostic des AIR surtout à la phase de début, en cas de trouble neurologique et dans la surveillance post-thérapeutique (Khalil et al., 2007). A Cotonou l'IRM n'est pas accessible à la majorité de la population et le scanner reste le seul recours imagerique pour la réalisation du bilan lésionnel des AIR et le suivit post-thérapeutique. Notre étude a permis de le corroborer. Mais néanmoins, les consultations tardives et le faible échantillonnage demeurent des biais qui ne pourrait amener à exclure la prescription de l'IRM dans l'exploration des AIR dans un contexte de favoriser.

\section{Conclusion}

$\mathrm{Au}$ terme de cette étude, dont l'objectif était de décrire les caractéristiques des infections rachidiennes à la TDM, au CNHU-HKM de Cotonou, il ressort que le diagnostic de ces infections a majoritairement été posé à la TDM avant la survenue de complications. Les spondylodiscites étaient plus fréquentes. Les érosions osseuses des plateaux vertébraux en miroir étaient fortement évocatrices en dehors de toute hypodensité discale. Cependant la tomodensitométrie a ses limites dans le diagnostic précoce des 
spondylodiscites. Il urge donc qu'une unité fonctionnelle d'IRM soit rapidement mise en place pour l'amélioration des performances diagnostiques

\section{References:}

1. Annabi, H., Abdelkafi, M., \& Trabelsi, M. (2008). La tuberculose ostéo-articulaire. Tun Orthop., 1(1), 7-17.

2. Bouillot, L., Kayayan, H., \& Goiset, E. (2015). De la scoliose idiopathique au mal de Pott: Présentation d'une observation et discussion diagnostique. Archives de Pediatrie, 22(12), 1256-1259. https://doi.org/10.1016/j.arcped.2015.09.019

3. Gbané-Koné, M., Ouattara, B., Diomandé, M., Sessou, V., Kaboré, F., Djoko, K. F., Traoré, M., Eti, E., \& Kouakou, N. M. (2015). Contribution of computed tomography in the diagnosis of spinal tuberculosis about 496 cases in Abidjan. The Pan African Medical Journal, 20, 201. https://doi.org/10.11604/pamj.2015.20.201.5996

4. Khalil, M. R., Ouali Idrissi, M., Cherif Idrissi El Ganouni, N., Hiroual, M. R., Haddi, M., Ait Benali, S., Essadki, O., \& Ousehal, a. (2007). OA-WS-54 Apport de l'imagerie en coupe (TDM-IRM) dans le diagnostic de la spondilodiscite tuberculeuse : serie de 50 cas. Journal de Radiologie, 88(1), 1581. https://doi.org/10.1016/S02210363(07)81919-6

5. Kumar, Y., Gupta, N., Chhabra, A., Fukuda, T., Soni, N., \& Hayashi, D. (2017). Magnetic resonance imaging of bacterial and tuberculous spondylodiscitis with associated complications and non-infectious spinal pathology mimicking infections: a pictorial review. BMC Musculoskeletal Disorders, $18(1), \quad 1-10$. https://doi.org/10.1186/s12891-017-1608-z

6. L.Badaoui, G.Dabo, M.Sodqi, L.Marih, A.Oulad lahsen, A.Chakib, K. E. F. M. (2014). Spondylodiscites infectieuses : Aspects épidémiocliniques, paracliniques, thérapeutiques et évolutives. Revue Malienne d'Infectiologie et de Microbiologie, 3, 29-33.

7. Lamini, N. E. (2019). Aspects morphologiques inhabituels du mal de pott chez l' adulte Unusual morphological aspects of pott 's disease. 11(numéro 3), 344-347.

8. Toloba Y, Diallo S, Maiga Y, Sissoko BF, Ouattara K, Soumare D, S. S. (2011). Tuberculose vertébrale (mal de pott) : aspects épidémio clinique, radiologique et évolutif au chu du point-G. Mali Médical, 26(2), 8-11.

9. Tyrrell P, C.-P. V. ., \& Mccall, I. W. (1999). Musculoskeletal radiology Review article Spinal infection. European Radiology, 1077(9), 1066-1077.

10. Y. Thouvenin, \& Cyteval, C. (2010). Imagerie des spondylodiscites 
infectieuses. La Lettre Du Rhumatologue, 362, 20-24.

11. Zomalheto, Z., Kobelembi, A., Agbodande, A., Zossoungbo, F., \& Avimadje, M. (2015). Profil épidémio-clinique, paraclinique et thérapeutique des spondylodiscites à germes banals en consultation hospitalière à Cotonou (Bénin ). Médecine d'Afrique Noire, 62(8/9), 405-410.

\section{Iconographie}

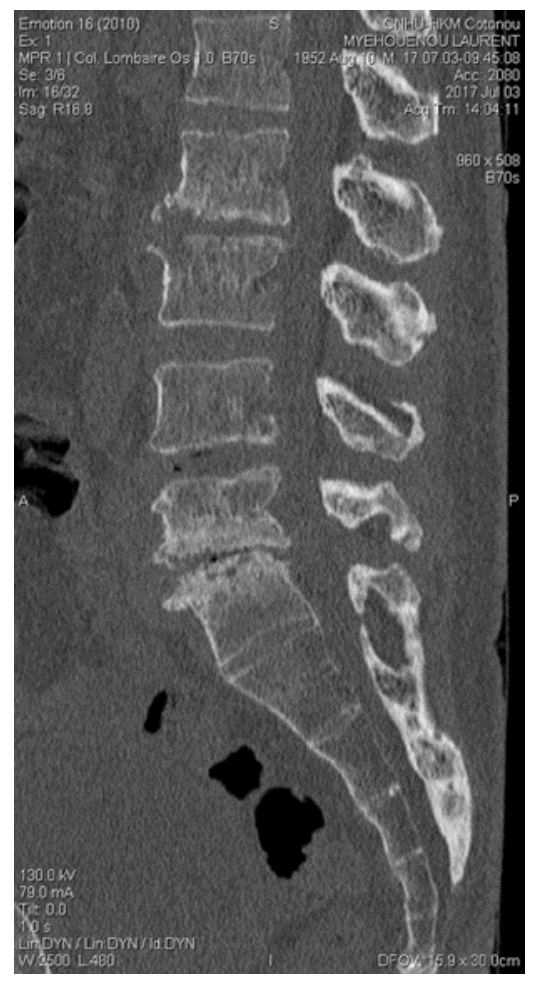

Figure 4 : Scanner du rachis lombaire en reconstruction sagittale en fenêtre osseuse : (a) Érosion en miroir de l'étage L1-L2 avec séquestre osseux en antérieur (AIR); (b) ostéocondensation en miroir avec pincement sévère de l'étage L4-L5 (lésions dégénératives) 


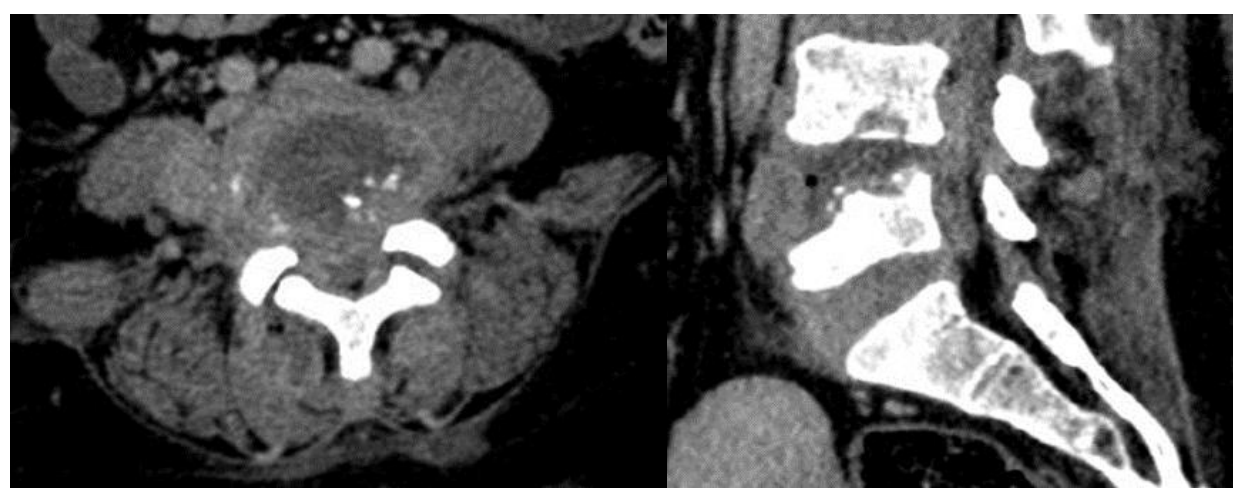

Figure 5 : Scanner du rachis lombaire, fenêtre discale en coupe axiale (a) et reconstruction sagittale(b) : une hypodensité du disque L4-L5, avec calcifications intra discales, un débord discal postérieur diffus et une compression médullaire. On retrouve un tassement cunéiforme de la vertèbre sous-jacente 